Check for updates

Cite this: RSC Adv., 2017, 7, 37467

Received 27th March 2017

Accepted 17th July 2017

DOI: 10.1039/c7ra03527f

rsc.li/rsc-advances

\section{Color conversion of the magnetically separable Al/ Fe oxide RNGO in the selective oxidation of benzyl alcohol induced the observation of its morphology change $\uparrow$}

\begin{abstract}
Qichen Li, Lingling Zhang, Xiaozhong Wang, Gang Xu, * Yingqi Chen and Liyan Dai (D)*
Herein, via integrating the merits of Fe-based nanoparticles and graphene oxide, magnetic $\mathrm{Al} / \mathrm{Fe}$ oxide nanoparticles implanted on reduced $\mathrm{N}$-doped graphene oxide (Al/Fe oxide RNGO) were synthesized by solvothermal reaction followed by thermal annealing with a particular temperature and hold time. The synthesized catalyst exhibited excellent performance with more than $80 \%$ yield of the desired product benzaldehyde achieved within a 2-hour reaction time. Due to its distinctive magnetism, the material can easily be separated and recycled for subsequent runs. It also demonstrated exceptional stability and recyclability with regard to its performance in a lifetime test without significant capacity or activity loss. The most remarkable finding was the observation of a color shift during the lifetime test due to the change in the morphology, which was further connected with the catalytic performance; initially, a chaotic structure and a comparable inferior catalytic activity were obtained, whereas a waffle or pastry state and a relatively superior activity were observed later. An additional reference without reactants did not display this morphology change; this result supported the employment of the catalyst in the reaction and provided a new alternative methodology to deduce and verify the mechanism.
\end{abstract}

\section{Introduction}

Nanoparticles or nanocrystals made of metals or oxides are of particular interest due to their mechanical, electrical, magnetic, optical, chemical, and other properties..$^{1-3}$ Since nanomaterials are being developed, the environmental and human health impacts of both the products and processes used to make them must be considered to ensure their long term economic viability. However, in this regard, the introduction of magnetic nanoparticles has tackled the above mentioned problem and made a splendid contribution to the progression of the chemistry synthesis. The potential and versatility of these small magnetic nanoparticles have streamlined the tedious and costly separation processes, which has always been a difficult challenge in chemistry. More significantly, the emergence of nanoparticles has exerted a visible effect on the synthesis of benzaldehyde. ${ }^{4-7}$

Benzaldehyde, the simplest aromatic aldehyde, is one of the most industrially versatile intermediates. It is a precursor of high demand in various domains e.g. in pharmaceuticals,

Zhejiang Provincial Key Laboratory of Advanced Chemical Engineering Manufacture Technology, College of Chemical and Biological Engineering, Zhejiang University, Hangzhou 310027, P. R. China. E-mail: dailiyan@zju.edu.cn; xugang_1030@zju. edu.cn; Fax: +86 571 87952394; Tel: +8657187952693

$\dagger$ Electronic supplementary information (ESI) available. See DOI: 10.1039/c7ra03527f cosmetics, pesticides, and herbicides, and some other fine chemicals. $^{8-17}$ The selective oxidation of primary alcohol is a key transformation from both synthetic and industrial points of view. ${ }^{18}$ This main synthetic method for obtaining carbonyl compounds has been a hit and has attracted significant attention.

The conventional method is basically carried out by utilizing stoichiometric amounts of high valence transition metal salts, such as dichromate, chromium trioxide or tungsten, which have been employed for many decades. ${ }^{19}$ The catalyst system based on tungsten that mediates the oxidation of alcohol can be traced to the pioneering study reported by Jacobson et al. in $1976 .^{20}$ Sometimes, even organic oxidants, such as the Swern ${ }^{21}$ and Dess-Martin ${ }^{22}$ reagents, have also been considered as a favorable alternative until the introduction and adoption of environmentally benign hydrogen peroxide.

The application of $\mathrm{H}_{2} \mathrm{O}_{2}$ suddenly intrigued extensive investigations of this seemingly simple and straightforward conversion due to its one-step procedure; however, this strategy actually turned out to be complicated and difficult. In previous studies, chemists have made several attempts to explore suitable conditions for catalyzing the selective oxidation of benzyl alcohol using this environmentally friendly reagent, but still failed in their endeavour. However, failures and trials offered some recognition and insight into making a progress. ${ }^{23 a-j}$ For most catalytic systems using $\mathrm{H}_{2} \mathrm{O}_{2}$, noble metals are 
inevitable. ${ }^{24}$ They have the significant advantage of extraordinary efficiency and specialized selectivity over their counterparts. However, either due to their high cost or the reason that noble metal residues jeopardize the use of produced intermediates in pharmaceutical and nutritional products, they are minimally utilized at the industrial scale..$^{25-28}$ Subsequently, cheap, transition metal-catalyzed oxidation of corresponding alcohols in the presence of $\mathrm{H}_{2} \mathrm{O}_{2}$ has been greatly explored. Moreover, it attracted significant attention from our group; thus, we aimed to develop readily available, economical, and more efficacious alternatives to traditionally employed gold or platinum in several catalytic reactions. Some homogenous and heterogeneous catalytic systems have been well investigated for $\mathrm{H}_{2} \mathrm{O}_{2}$ activation; Zauche et al. have reported that $\mathrm{Re}$ is an ideal catalytic active constituent. $^{29}$ Recently, the Liu Group reported palladiumcatalysed selective oxidation of alcohol and achieved a fairly excellent yield.$^{30}$ Manganese, ruthenium, tin, iron, and copper ${ }^{31-37}$ have also been proved to be perfect options in this conversion. Among these transition metals, iron, however, has numerously been explored not only for its availability at a low cost, but also for its notable stability and remarkable dispersibility on supports. $^{38-42}$ However, most catalysts employing iron still demonstrated an unsatisfactory and undesirable performance with regard to long reaction times and demanding conditions.

Recently, the emergence of graphene (first isolated in 2004 by Andre Geim and Konstantin) made it possible to quantify the promotion of catalytic performance. High quality graphene with size in hundreds of microns allowed the investigation of their amazing properties. Excellent transmittance and superior intrinsic carrier mobility aroused several attempts to use graphene as a catalyst support. Basically, graphene oxide and reduced graphene oxide or sometime N, B, S or other elementdoped graphene oxide is actually used as a host. To our surprise and delight, most catalysts exhibited better performance even with the substitution of the host by graphene, without changing the active center. Therefore, it has always been regarded as a superior carrier. ${ }^{43 a-e}$

In this regard, we tailored magnetic $\mathrm{Al} / \mathrm{Fe}$ oxides implanted on reduced $\mathrm{N}$-doped graphene oxide (Al/Fe oxide RNGO), which exhibited excellent performance with a desirable product yield of more than $80 \%$ within a 2 hour reaction during its application in the conversion of benzaldehyde. In addition, we characterized the catalyst before and after 10 runs. We discovered a peculiar phenomenon: the morphological change in the catalyst. A remarkable observation was that a designed blank comparative experiment showed no morphology alterations, testifying that the employment of the catalyst in the reaction also provided an alternative methodology to deduce and verify the mechanism of the reaction.

\section{Experimental}

\section{Chemicals}

All chemicals used were of analytical grade and used as received from Sinopharm Chemical Reagent Co., Ltd without any purification. Ultra-pure water was prepared in the laboratory using a Milli-Q SP reagent water system from Millipore (Milford, MA).

\section{Synthesis of $\mathrm{Al} / \mathrm{Fe}$ oxide RNGO}

$\mathrm{Al} / \mathrm{Fe}$ oxide RNGO was synthesized via the solvothermal process by pyrolysis of graphene oxide (GO) using a N-source and corresponding metal salt. GO was prepared via a modified Hummers method and subsequent exfoliation. ${ }^{44}$ In the preparation, sulfuric acid $(98 \%, 78 \mathrm{~mL})$ was slowly added to a mixture of graphite powder $(3.0 \mathrm{~g})$ and $\mathrm{NaNO}_{3}(1.2 \mathrm{~g})$; then, the mixture was cooled down to $0{ }^{\circ} \mathrm{C}$ in an ice bath. $\mathrm{KMnO}_{4}(12 \mathrm{~g})$ was added after $30 \mathrm{~min}$ and the mixture was stirred for $60 \mathrm{~min}$ at $<20^{\circ} \mathrm{C}$. Then, the solution was heated to $35^{\circ} \mathrm{C}$ and stirred for $90 \mathrm{~min}$, during which deionized water $(138 \mathrm{~mL})$ was gradually added. The temperature was maintained at $98{ }^{\circ} \mathrm{C}$ for $8 \mathrm{~min}$. Additional water $(300 \mathrm{~mL})$ and $\mathrm{H}_{2} \mathrm{O}_{2}(30 \%, 10 \mathrm{~mL})$ were added after air cooling for $15 \mathrm{~min}$. The resulting solution was washed to neutral via centrifugation with the $\mathrm{HCl}$ solution (5\%) and water to remove chloride and sulfate ions. The graphite oxide slurry was then freeze dried for $48 \mathrm{~h}$. Then, $0.12 \mathrm{~g}$ pre-synthesized GO was delaminated by treating in an ultrasonic equipment for $2 \mathrm{~h}$. This was then mixed with equal molar amounts of $\mathrm{Al}\left(\mathrm{NO}_{3}\right)_{3}$ $\cdot 9 \mathrm{H}_{2} \mathrm{O}(4 \mathrm{mmol})$ and $\mathrm{FeCl}_{3} \cdot 6 \mathrm{H}_{2} \mathrm{O}(4 \mathrm{mmol})$ and $1.3 \mathrm{~g}$ urea with ethylene glycol as the dispersion medium and vigorously stirred for $2 \mathrm{~h}$. When it was homo-dispersed, $2 \mathrm{~mL}$ of 1-methyl-2pyrrolidone was added to the suspension, which was then immediately transferred to a Teflon-lined autoclave for the hydrothermal reaction at $180{ }^{\circ} \mathrm{C}$ for time $12 \mathrm{~h}$. The catalyst was obtained by washing the abovementioned suspension with ethanol and deionized water via centrifugation, drying at $50{ }^{\circ} \mathrm{C}$ for $24 \mathrm{~h}$, and further thermally treating by placing the catalyst into a quartz boat and heating to $500{ }^{\circ} \mathrm{C}$ for $2 \mathrm{~h}$ under a $\mathrm{N}_{2}$ atmosphere at the heating rate of $10{ }^{\circ} \mathrm{C} \mathrm{min}^{-1}$. After the furnace was normally cooled to room temperature, the final product was obtained. A variety of catalysts treated under different thermal annealing conditions were also prepared for further comparison of their catalytic activities and to obtain better insights about the influence that thermal annealing exerted on the catalysts.

\section{Catalytic activity test}

The selective oxidation of benzoic alcohol with $30 \%$ hydrogen peroxide as the oxidizing agent was carried out to test the catalytic activity of the prepared $\mathrm{Al} / \mathrm{Fe}$ oxide RNGO. All experiments were conducted in a reaction tube with $1 \mathrm{mmol}$ benzyl alcohol, hydrogen peroxide, which was added every $30 \mathrm{~min}$ with a dosage of $40 \mu \mathrm{L}$ ( 4 times), and $2 \mathrm{~mL}$ acetonitrile as the solvent. In addition, we conducted a series of experiments to optimize the reaction conditions such as temperature, time, the dosage of $\mathrm{H}_{2} \mathrm{O}_{2}$ and catalyst, and the solvent. The product was separated and filtered from the reaction tube by applying a magnet to the outside, trapping, and immobilizing the catalyst. After this, the reaction products were analyzed and tested by HPLC with a PDA-100 photodiode array detector, and an Acclaim $120 \mathrm{C} 18$ column $(5 \mu \mathrm{m}, 4.6 \times$ $250 \mathrm{~mm}$ ) was used to determine the concentration of benzaldehyde. The mobile phase was composed of methanol and water $(90: 10, \mathrm{v} / \mathrm{v})$ at a flow rate of $1.0 \mathrm{~mL} \mathrm{~min}^{-1}$ at a column temperature of $40{ }^{\circ} \mathrm{C}$. 


\section{Characterization}

The morphologies of the synthesized Al/Fe oxide RNGO were surveyed using a JEM-2010 transmission electron microscope with an energy dispersive X-ray spectrometer (TEM-EDX, JEOL, Japan) and scanning electronic microscope (SEM, Hitachi SU8020, Japan). X-ray diffraction studies (XRD, PANalytical X' Pert diffractometer, Almelo, Netherlands) were performed to characterize the crystal form using a monochromatized X-ray beam with nickel-filtered $\mathrm{Cu} \mathrm{K} \alpha$ radiation at a $0.4^{\circ} \mathrm{min}^{-1}$ scan rate. Inductively coupled plasma mass spectrometry (ICPMS) was adopted to analyze the composition and ratio of the elements.

\section{Results and discussion}

\section{Catalyst selection and catalytic performance}

A facile two-step solvothermal reaction followed by thermal annealing was utilized for the synthesis of $\mathrm{Al} / \mathrm{Fe}$ oxide $\mathrm{RNGO}$ with a range of different thermal annealing temperatures and hold times. From Fig. 1(a), it could be concluded that the yield of benzaldehyde basically increased and then decreased and finally peaked at $500{ }^{\circ} \mathrm{C}$, and a $78.9 \%$ yield with the variation of temperature was obtained. Moreover, the same tendency was observed for the time variable, as shown in Fig. 1(b), and the maximal yield was obtained at 4 hour thermal treatment. In addition, as the thermal treatment time increases, the selectivity of benzaldehyde gradually decreases with the increasing percentage of benzoic acid.

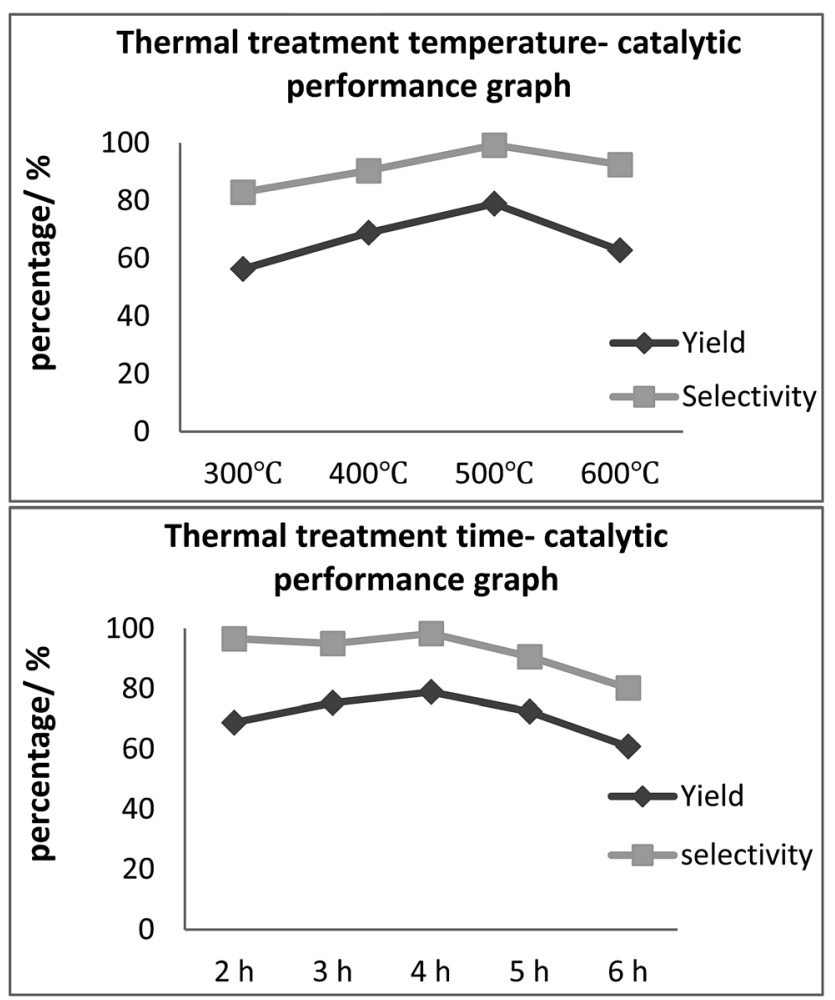

Fig. 1 The catalytic performance of the thermally treated Al/Fe oxide RNGO at different temperatures and times.
To achieve the best catalytic performance, we also optimized the reaction parameters such as the reaction time, reaction temperature, hydrogen peroxide dosage, and catalyst loading. From Fig. 2(a), it can be concluded that the optimal loading of $\mathrm{Al} / \mathrm{Fe}$ oxide $\mathrm{RNGO}$ is $10 \%$ (mol) under the typically identical conditions abovementioned in the Experimental section. Lower loading of the catalyst cannot effectively accelerate the decomposition of $\mathrm{H}_{2} \mathrm{O}_{2}$, whereas higher loading otherwise leads to the quicker generation of $\mathrm{OH}$ and over oxidation of the intermediate and thus the larger proportion of benzoic acid.

The dosage of $\mathrm{H}_{2} \mathrm{O}_{2}$ was investigated in a similar way. The best dosage was $40 \mu \mathrm{L}$ in one time for every 30 min injection, which was concluded from Fig. 2(b). Although the conversion of benzyl alcohol increased as the $\mathrm{H}_{2} \mathrm{O}_{2}$ ratio increased, the selectivity to benzaldehyde decreased. As a result, the obtained product would contain more benzoic acid, which could even be the major product at a certain $\mathrm{H}_{2} \mathrm{O}_{2}$ dosage value. Therefore, the best dosage of $\mathrm{H}_{2} \mathrm{O}_{2}$ was chosen to be $40 \mu \mathrm{L}$ from this experiment.

Reaction temperature has a dramatic effect on the catalytic performance of selective oxidation. Thus, the temperature condition is an essential index for a catalyst. The temperature range was investigated from $20{ }^{\circ} \mathrm{C}$ to $100{ }^{\circ} \mathrm{C}$. The best temperature setting was $80^{\circ} \mathrm{C}$. According to Fig. 2 (c), although $90^{\circ} \mathrm{C}$ or even $100{ }^{\circ} \mathrm{C}$ may exhibit a better performance in the reaction with the regard to the yield of benzaldehyde, the boiling point of acetonitrile is $82{ }^{\circ} \mathrm{C}$, which, as a solvent, hampers the reaction to a certain degree. Moreover, the increase in temperature cannot improve the yield greatly; we finally decided the best reaction temperature to be $80{ }^{\circ} \mathrm{C}$, considering energy consumption in the future industrial scale application.

To investigate the effect of reaction time, $160 \mu \mathrm{L} \mathrm{H}_{2} \mathrm{O}_{2}$ was added in one step instead of four, as described in the
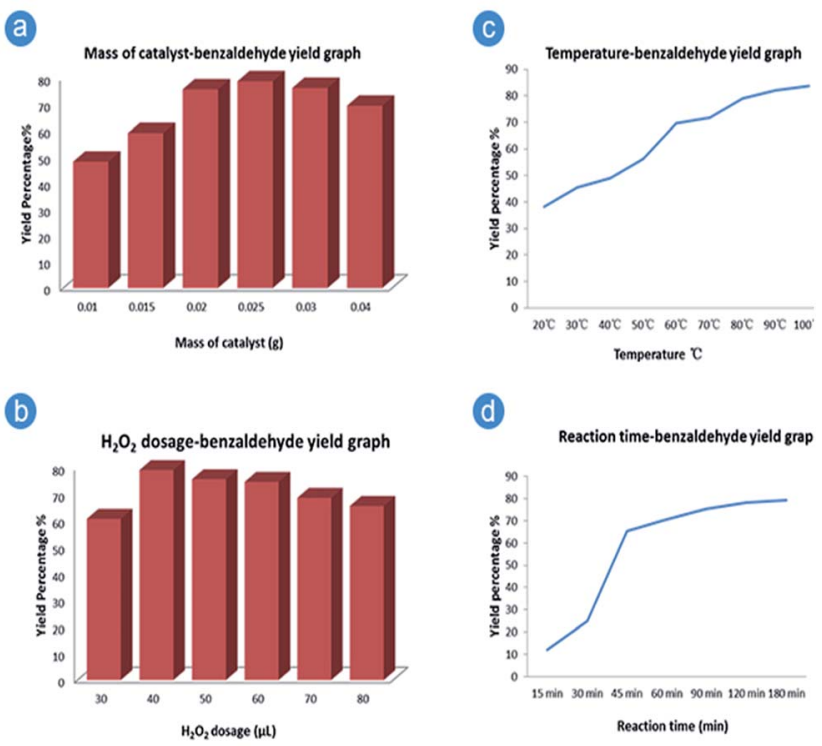

Fig. 2 Effects of reaction parameters on the catalytic performance of $\mathrm{Al} / \mathrm{Fe}$ oxide RNGO. (a) Catalyst loading; (b) $\mathrm{H}_{2} \mathrm{O}_{2}$ loading; (c) reaction temperature; and (d) reaction time. 


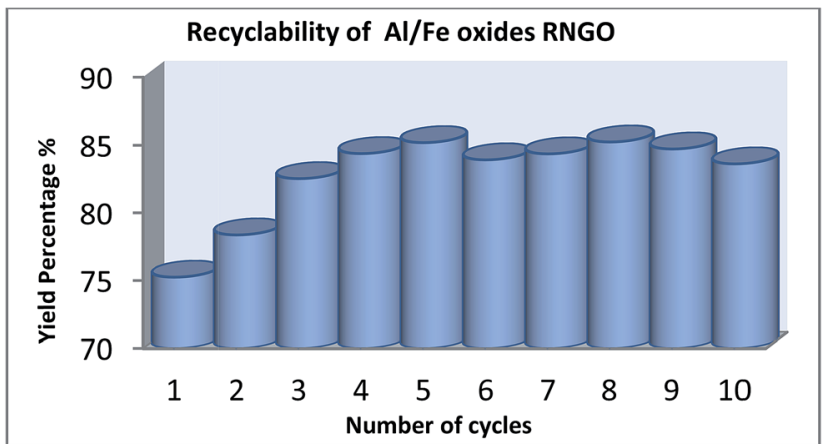

Fig. 3 Recyclability of Al/Fe oxide RNGO. The recovered catalyst was readily used in the next run without any further treatment.

experimental section. It should be noted that the addition of $\mathrm{H}_{2} \mathrm{O}_{2}$ in one step led to a lower yield. The result reveals that there is an induction period at the initial stage of the reaction. Thereafter, the reaction accelerated until it was limited due to the decreasing concentration of $\mathrm{H}_{2} \mathrm{O}_{2}$. The yield of the product was still increasing after the fast increase between $30 \mathrm{~min}$ and 45 min. According to Fig. 2(d), the optimum reaction time should be 2 hours.

$\mathrm{Al} / \mathrm{Fe}$ oxide RNGO exhibited favourable magnetic properties. We tested its reversibility by stabilizing the catalyst with an external magnet, followed by filtration and then using HPLC to analyze the yield of the reaction. As observed from Fig. 3, it showed a steady increase in yield in the first three runs and maintained its prime performance in the following runs without significant drop. According to most previous similar studies, it should suffer a loss during its deployment in the reaction. However, in our research, an opposite trend was obtained. Moreover, we also observed some peculiar phenomenon, which also was the most interesting part: the color of the catalyst changed with its catalytic performance. It gradually turned to brick red from somewhat black during its application in the reaction in the first several runs and then retained the

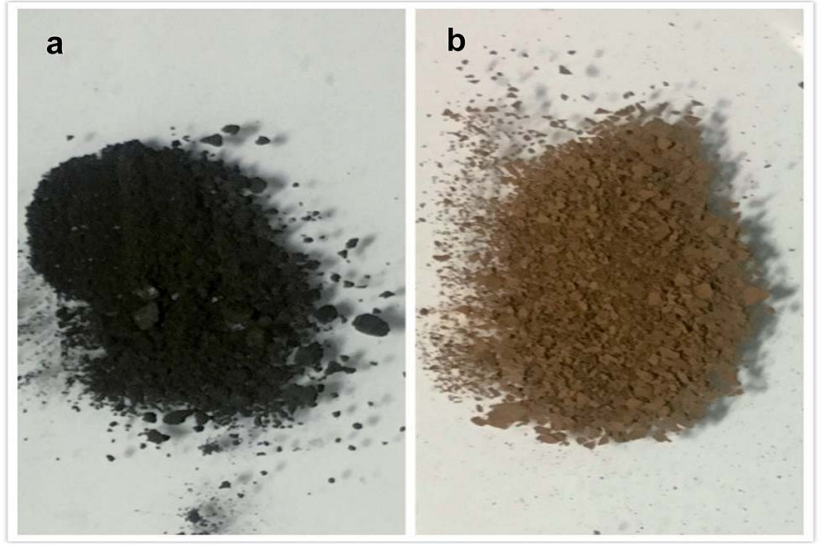

Fig. 4 The color change during the recycling. (a) The initial color before its application in the selective oxidation reaction; (b) the color of catalyst after the fifth cycle.

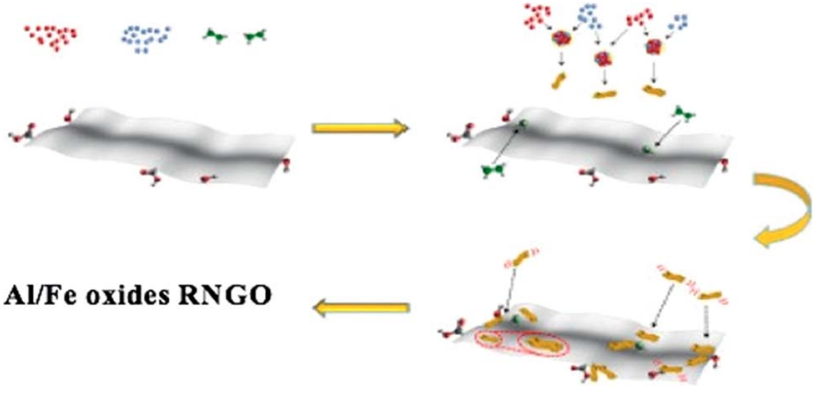

Fig. 5 Schematics of the preparation of Al/Fe oxide RNGO.

color without turning back (Fig. 4). The catalytic performance on the other hand, reflected from the yield of benzaldehyde, became better and better in the first subsequent runs and the prime performance was maintained without significant loss in the next trials.

\section{Physicochemical properties of the material}

Based on several previous studies on the morphology and structural features of the catalyst, we proposed a possible formation mechanism of these different types of nanohybrids, as schematically illustrated in Fig. $5{ }^{45 a-c}$ Upon the first sonication, the interweaving of $\mathrm{Al}^{3+}$ and $\mathrm{Fe}^{3+}$ cations with ethylene glycol molecules formed nanoscale clusters. Under a solvothermal reaction, nanoclusters started to nucleate and formed regular granules due to their tendency to randomly aggregate. Further annealing led to the formation of a chaotically arranged nanosheet. The N-doped RGO skeletons served as superior templates for the growth and formation of nanosheets of mixed transition metal oxides. However, due to the weak bonding between $\mathrm{N}$-doped RGO and transition metal oxides, a few RGO sheets remained uncoated.

Structural and morphological analyses of the prepared catalysts were conducted via SEM and HRTEM. The SEM images

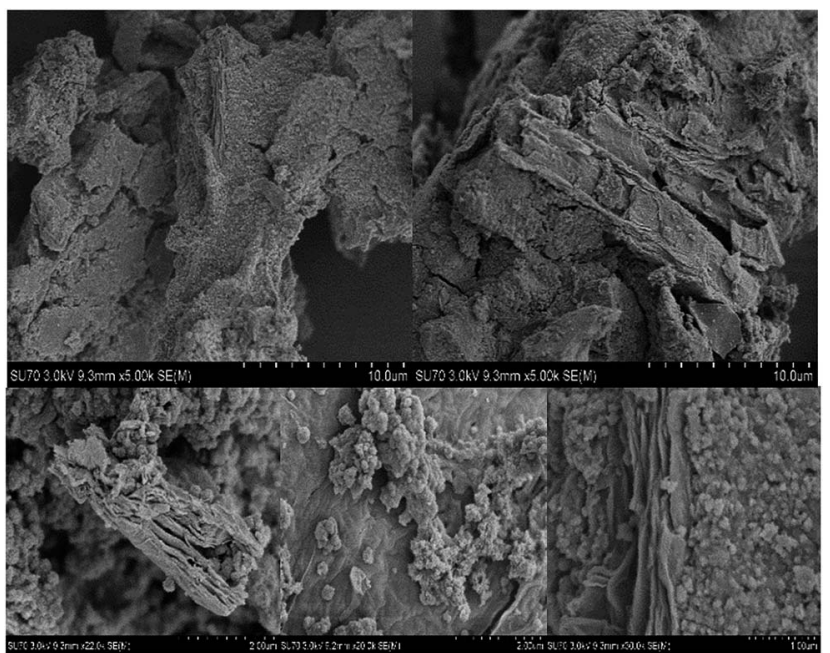

Fig. 6 SEM images of Al/Fe oxide RNGO before its application in the reaction. 


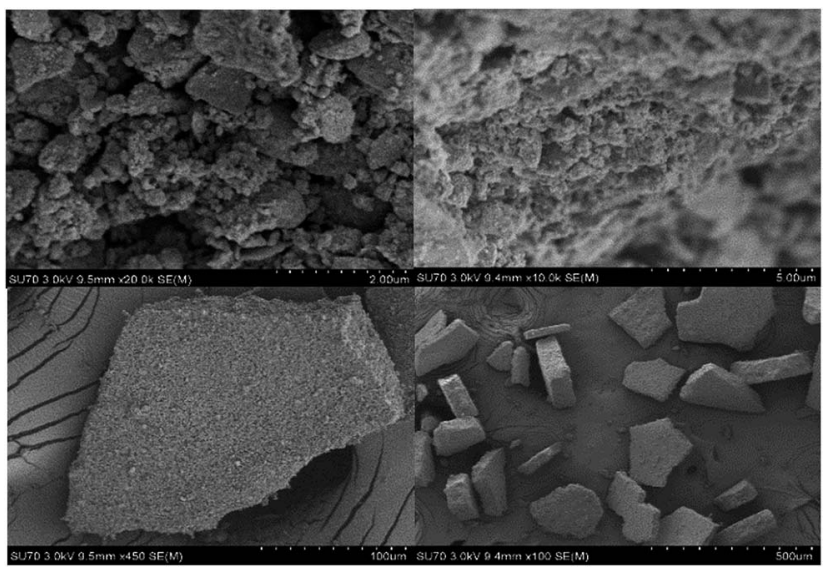

Fig. 7 SEM images of $\mathrm{Al} / \mathrm{Fe}$ oxide RNGO after its fifth run in the recyclability experiment.

of $\mathrm{Al} / \mathrm{Fe}$ oxide $\mathrm{RNGO}$ in Fig. 6 demonstrate the chaotic morphology as a whole. When the images were magnified, we found a structure that was similar to that we proposed, with one layer and dotted with metal oxide nanoparticles in some parts of the sheet and with multiple layers stacked together in a disarranged way. These closely packed nanoparticles anchored onto the surface of the N-doped RGO sheet are essential for the selective oxidation of benzyl alcohol. ${ }^{46}$ As we have mentioned in the recyclability experiment, the color change during the application of the catalyst has attracted our attention. Thus, we further examined the structure of the catalyst after the fifth run. To our surprise, we found a shift in the aggregation state from a chaotic type to a regular waffle or pastry state (Fig. 7); this explained the possible reason for the color conversion. The vigorous stirring transformed the catalyst into single nanosheets from the initially disordered, piled up form. These
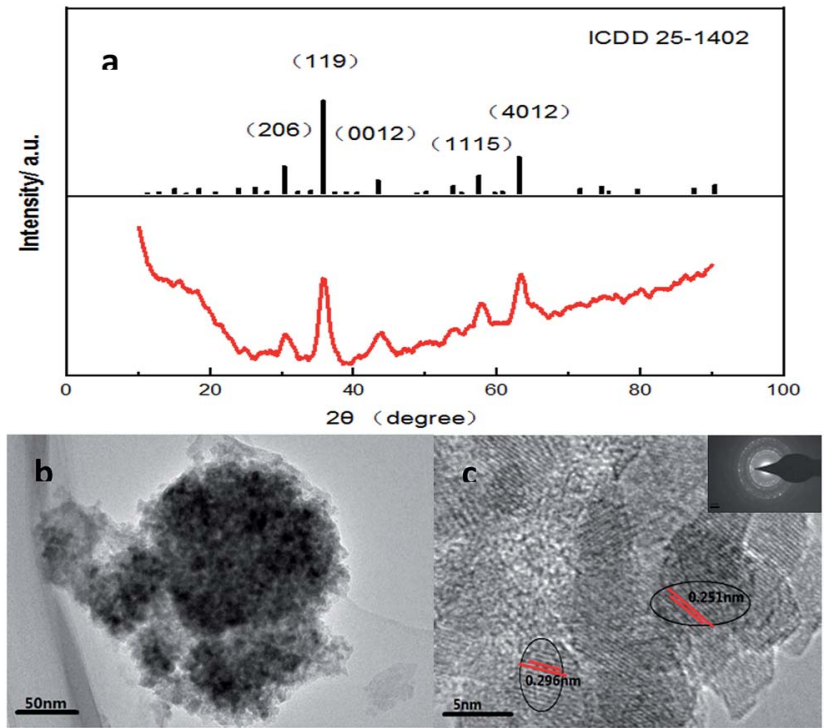

Fig. 8 (a) XRD pattern with the peak positions in the ICDD card; (b) FETEM image; and (c) HRTEM image and its Fourier transform (inset). nanosheets dotted with metal oxides reacted with the reactants. After the reaction, because of the inherent magnetism, these nanosheets would reunite in a favorable way, thus causing the shift in the morphology. To validate our hypothesis, we carried out an extra blank experiment under the same condition as the those of the recyclability experiment but with no reactant to show if the same observation would occur. To our delight, neither a color change nor a morphology change was detected. This observation proved the involvement of the catalyst in the reaction. It indicated that the optimal performance was also achieved when the nanosheets were arranged in a regular waffle or pastry state.

All the peaks in the XRD pattern were assigned to maghemite, basically known as $\gamma-\mathrm{Fe}_{2} \mathrm{O}_{3}$ having a nutty structure in a chaotic aggregation state (Fig. 8(a)). On the basis of the ICPMS and EDX analysis, the catalyst prepared loaded $\mathrm{Al}$ oxides as well, and the amount of loading was nearly with the same with $\mathrm{Fe}$, slightly higher to be exactly. According to the EDX results, the percentages of the elements $\mathrm{Al}, \mathrm{Fe}$, and $\mathrm{O}$ are 6.90, 4.26, and 45.30, respectively, in one spot and 7.84, 5.23, and 56.96 in another. However, no Al oxides ICDD card perfectly matched. Corundum structure is the closest match with the same shift when $2 \theta$ is 35.136 and 66.546. Perhaps, the loaded Al oxides possessed different crystalline structures and dispersed in a random way with the corundum structure forming the majority. Based on the SEM test results, we can further confirm the color conversion hypothesis we proposed during the observation of morphology change. The HRTEM image showed
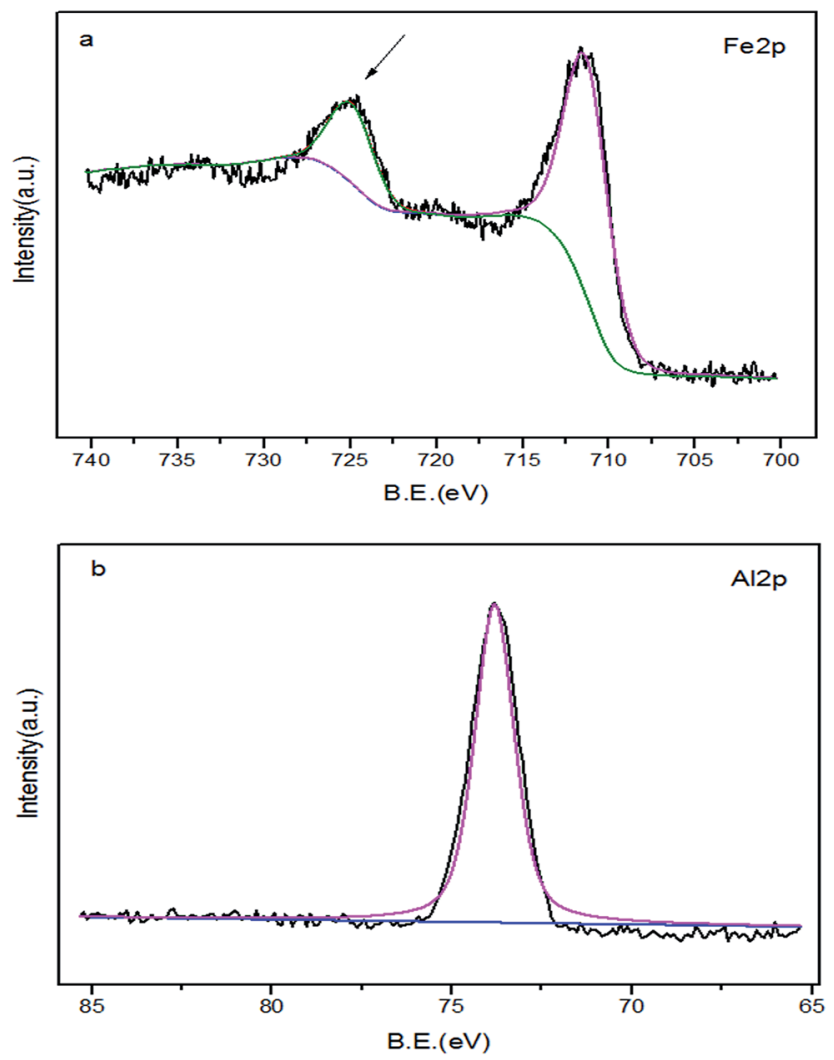

Fig. 9 XPS spectra of the catalyst. (a) Fe $2 p$ and (b) Al 2p. 

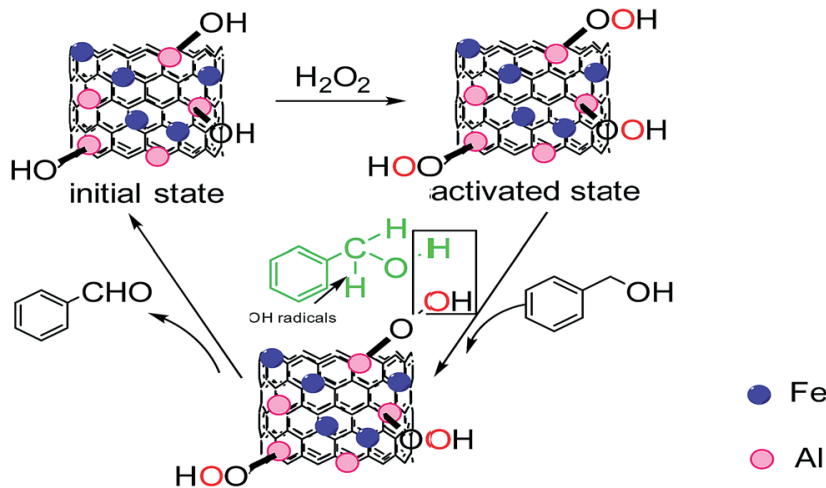

$\bigcirc \mathrm{Al}$

Fig. 10 The proposed mechanism for reaction over $\mathrm{Al} / \mathrm{Fe}$ oxides RNGO.

the lattice fringe of $0.251 \mathrm{~nm}$ and $0.296 \mathrm{~nm}$ corresponding to the (119) plane and (206) plane (Fig. 8(c)).

Additional qualitative evidence for the installation of surfacebound amide groups was obtained through X-ray photoelectron spectroscopy (XPS) analysis of the prepared catalyst. The spectra confirmed the presence of $\mathrm{Fe}$ and $\mathrm{Al}$ elements. High resolution XPS spectra in the region of the Fe 2p core level (Fig. 9(a)) indicated that the $\mathrm{Fe} 2 \mathrm{p}_{3 / 2}$ levels of magnetite had similar BE values of the $\mathrm{Fe}_{3} \mathrm{O}_{4}$ particles at $c a .710 .5 \mathrm{eV}$. The $\mathrm{Fe} 2 \mathrm{p}_{3 / 2} \mathrm{BE}$ of maghemite $\left(\gamma-\mathrm{Fe}_{2} \mathrm{O}_{3}\right)$ or hematite $\left(\alpha-\mathrm{Fe}_{2} \mathrm{O}_{3}\right)$ is only $0.4 \mathrm{eV}$ higher than that of magnetite, which may interfere with the assignment of the surface phase as magnetite. However, we observed a characteristic shake-up satellite of maghemite or hematite in these spectra, which should otherwise appear at $c a .8 .0 \mathrm{eV}$ above the $\mathrm{Fe} 2 \mathrm{p}_{3 / 2}$ peak (indicated by the arrow in Fig. 9(a)). The intensity of the shake-up satellite originated predominantly from the coupling of the iron core-hole state with the valence band and turned out to be a highly reliable indicator to distinguish the surface phase on iron oxides. ${ }^{47}$

\section{Mechanism}

The proposed mechanism for the reaction over $\mathrm{Al} / \mathrm{Fe}$ oxide RNGO is shown in Fig. 10. The initial state of the catalyst mainly contains $\mathrm{Fe}^{3+}$ and $\mathrm{Al}-\mathrm{OH}$ groups. After the induction period (about $0.5 \mathrm{~h}$ ), the active species $\mathrm{Fe}^{2+}$ and $\mathrm{Al}-\mathrm{OOH}$ are formed via interaction with $\mathrm{H}_{2} \mathrm{O}_{2}$. BzOH interacts with $\mathrm{Al}-\mathrm{OOH}$ to form an active surface complex and $\mathrm{H}_{2} \mathrm{O}_{2}$ is decomposed at the adjacent $\mathrm{Fe}^{2+}$ site to generate $\mathrm{OH}$ radicals. Surface-bonded $\mathrm{BzOH}$ is then selectively oxidized to benzaldehyde via simultaneous abstraction of two hydrogen atoms: one from the methylene group by $\mathrm{OH}$ radicals and the other from the hydroxyl group by Al-OOH. It is in this step that the synergistic effect between Fe and $\mathrm{Al}$ active sites may be involved. The catalytic cycle completes after the benzaldehyde desorption, which restores the initial state of the catalyst surface.

\section{Conclusions}

In conclusion, a facile method has been developed to fabricate novel Al/Fe oxide RNGO with $\gamma-\mathrm{Fe}_{2} \mathrm{O}_{3}$ NPs and multiple types of
Al oxides NPs (mostly corundum) embedded in the RNGO matrix via solvothermal reaction followed by a particular thermal annealing process. The prepared catalyst demonstrated superior catalytic activity and recyclability toward the selective oxidation of benzyl alcohol owing to its enrichment ability and abundant active sites. The most important part of this research is the finding of the morphology change in accordance with the catalytic performance through the observation of color shift during the lifetime test. Initially, a chaotic structure implied comparably inferior catalytic activity, whereas a waffle or pastry state was accompanied by relatively superior activity at peak performance. It supported the use of the catalyst in the reaction and helped in the verification of the most heterogeneous catalytic reaction.

\section{Acknowledgements}

This work was supported by the NSFC (No. 21176213), and Zhejiang Key Innovation Team of Green Pharmaceutical Technology (No. 2010R50043).

\section{Notes and references}

1 C. Stephenson and A. Hubler, Sci. Rep., 2015, 5, 15044.

2 A. Hubler and D. Lyon, IEEE Trans. Dielectr. Electr. Insul., 2013, 20, 1467-1471.

3 P. Kerativitayanan, J. K. Carrow and A. K. Gaharwar, Adv. Healthcare Mater., 2015, 4, 1600-1627.

4 G. Zhan, J. Huang, M. Du, D. Sun, I. Abdul-Rauf, W. Lin, Y. Hong and Q. Li, Chem. Eng. J., 2012, 187, 633-641.

5 N. Long and N. Quan, React. Kinet., Mech. Catal., 2015, 114, 147-155.

6 F. Shi, M. K. Tse, M. M. Pohl, A. Bruckner, S. Zhang and M. Beller, Angew. Chem., Int. Ed., 2007, 46, 8866-8868.

7 C. Kamonsatikul, T. Khamnaen, P. Phiriyawirut, S. Charoenchaidet and E. Somsook, Catal. Commun., 2012, 26, 1-5.

8 R. C. Larock, Comprehensive Organic Transformations, 1989, pp. 604-834.

9 R. A. Sheldon and J. K. Koshi, Metal-catalyzed Oxidations of Organic Compounds, 1981, vol. 2, pp. 68-73.

10 R. W. Dugger, J. A. Ragan and D. H. B. Ripin, Org. Process Res. Dev., 2005, 9, 253-258.

11 J. S. Carey, D. Laffan, C. Thomson and M. T. Williams, Org. Biomol. Chem., 2006, 4, 2337-2347.

12 X. Wang, G. Wu, J. Li, N. Zhao, W. Wei and Y. Sun, Catal. Lett., 2007, 119, 87-94.

13 J. U. Ahmad, M. T. Raisanen, M. Leskela and T. Repo, Appl. Catal., A, 2012, 411-412, 180-187.

14 C. Ragupathi, J. Judith Vijaya, R. Thinesh Kumar and L. John Kennedy, J. Mol. Struct., 2015, 1079, 182-188.

15 V. Mahdavi, H. R. Hashneminasab and S. Abdollahi, J. Chin. Chem. Soc., 2010, 57, 189-198.

16 M. J. Beier, T. W. Hansen and J. D. Grunwaldt, J. Catal., 2009, 266, 320-330.

17 A. Jia, L. Lou, C. Zhang, Y. Zhang and S. Liu, J. Mol. Catal. A: Chem., 2009, 306, 123-129. 
18 B. Karimi, F. B. Rostami, M. Khorasani, D. Elhamifar and H. Vali, Tetrahedron, 2014, 70, 6114-6119.

19 S. V. Ley and A. Madfin, in Comprehensive Organic Synthesis, 1991, vol. 7, pp. 251-289.

20 S. E. Jacobson, F. Mares and D. A. Muccigarasso, J. Org. Chem., 1979, 44, 921-924.

21 A. J. Mancuso, S. L. Huang and D. Swern, J. Org. Chem., 1978, 12, 2480-2482.

22 D. B. Dess and J. C. Martin, J. Org. Chem., 1983, 48, 41554156.

23 (a) A. J. Pearson and Y. Kwak, Tetrahedron Lett., 2005, 46, 5417-5419; (b) B. McAteer, N. Beattie and D. T. Richens, Inorg. Chem. Commun., 2013, 35, 284-289; (c) Rezaeifard, M. Jafarpour and A. Naeimi, Catal. Commun., 2011, 16, 240-244; (d) E. Farnetti and C. Crotti, Catal. Commun., 2016, 84, 1-4; (e) G. J. Ligtenbarg, P. Oosting, G. Roelfes, R. M. La Crois, M. Lutz, A. L. Spek, R. Hage and B. L. Feringa, Chem. Commun., 2001, 385-386; (f) Wang, R. Liu, J. Chen and X. Liang, Chem. Commun., 2005, 53225324; (g) B. H. Lipshutz, M. Hageman, J. C. Fennewald, R. Linstadt, E. Slack and K. Voigtritter, Chem. Commun., 2014, 50, 11378-11381; (h) Minisci, C. Punta, F. Recupero, F. Fontana and G. Franco Pedulli, Chem. Commun., 2002, 688-689; (i) Shi, M. Kin Tse, Z. Li and M. Beller, Chem.Eur. J., 2008, 14, 8793-8797; (j) Yan, Y. C. Fang, Y. X. Jia and X. H. Duan, New J. Chem., 2017, 41, 2372-2377.

24 C. Parmeggiani and F. Cardona, Green Chem., 2012, 14, 547564.

25 D. Obermayer, A. M. Balu, A. A. Romero, W. Goessler, R. Luque and C. O. Kappe, Green Chem., 2013, 15, 1530-1537.

26 C. E. Garrett and K. Prasad, Adv. Synth. Catal., 2004, 346, 889-900.

27 C. J. Welch, J. Albaneze-Walker, W. R. Leonard, M. Biba, J. DaSilva, D. Henderson, B. Laing, D. J. Mathre, S. Spencer, X. Bu and T. Wang, Org. Process Res. Dev., 2005, 9, 198-205.

28 J. T. Bien, G. C. Lane and M. R. Oberholzer, Top. Organomet. Chem., 2004, 6, 263-268.

29 T. H. Zauche and E. H. James, Inorg. Chem., 1998, 37, 68276831.

30 J. Liu, F. Wang, K. Sun and X. Xu, Catal. Commun., 2008, 9, 386-390.
31 A. Berkessel and C. A. Skorz, Tetrahedron Lett., 1999, 44, 7965-7968.

32 H. R. Mardani and H. Golchoubian, Tetrahedron Lett., 2008, 47, 2349-2352.

33 Z. Ye, Z. Fu, S. Zhong, F. Xie, X. Zhou, F. Liu and D. Yin, J. Catal., 2009, 261, 110-115.

34 P. Saisaha, L. Buettner, R. Hage, B. L. Feringa, W. R. Browne and J. W. de Bauerm, Adv. Synth. Catal., 2013, 355, 25912603.

35 S. E. Martin and A. Garrone, Tetrahedron Lett., 2003, 44, 549552.

36 E. Balogh-Hergovicha and G. Speier, J. Mol. Catal. A: Chem., 2005, 230, 79-83.

37 M. Lenze and E. B. Bauer, Chem. Commun., 2013, 5889-5891. 38 H. Lim, J. Lee, S. Jin, J. Kim, J. Yoon and T. Hyeon, Chem. Commun., 2006, 463-465.

39 J. M. Campelo, D. Luna, R. Luque, J. M. Marinas and A. A. Romero, ChemSusChem, 2009, 2, 17-34.

40 A. Karmakar and L. M. D. R. S. Martins, Catal. Lett., 2015, 145, 2066-2076.

41 M. P. Krzeminski and A. Wojtcazk, Tetrahedron Lett., 2005, 46, 5417-5419.

42 N. Wang, R. Liu, J. Chen and X. Liang, Chem. Commun., 2005, 5322-5324.

43 (a) A. K. Geim, Science, 2009, 324(5934), 1530-1534; (b) A. K. Geim and K. S. Novoselov, Nat. Mater., 2007, 6(3), 183-191; (c) B. Guo, L. Fang, B. Zhang and J. Gong, Insci. J., 2011, 1(2), 80-89; (d) C. Huang, C. Li and G. Shi, Energy Environ. Sci., 2012, 5, 8848-8868; (e) V. Singh, D. Joung, L. Zhai, S. Das, S. I. Khondaker and S. Seal, Prog. Mater. Sci., 2011, 56, 1178-1271.

44 W. S. Hummers and R. E. Offeman, J. Am. Chem. Soc., 1958, 80, 1339.

45 (a) L. Hu, B. Qu, C. Li, Y. Chen, L. Mei, D. Lei, L. Chen, Q. Li and T. Wang, J. Mater. Chem. A, 2013, 1, 5596-5602; (b) S. Sahoo, S. H. Bae, Y. S. Lee, J. M. Lee, J. M. Ahn, C. G. Kim and I. K. Oh, Carbon, 2015, 94, 455-463; (c) Y. Zhan, J. Huang, Z. Lin, X. Yu, D. Zeng, X. Zhang, F. Xie, W. Zhang, J. Chen and H. Meng, Carbon, 2015, 95, 930-939.

46 K. Liu, T. Chen, Z. Hou, Y. Wang and L. Dai, Catal. Lett., 2014, 144, 314-319.

47 Y. Zhu, L. Tian, Z. Jiang, Y. Wei, S. Xie and M. Qiao, J. Catal., 2011, 281, 106-118. 